\title{
Gerencia del capital intelectual
}

\author{
Intelectual capital management
}

\author{
Daniel Arturo Sanz Del Vecchio** \\ Tito José Crissien Borrero**
}

\section{RESUMEN}

El propósito del presente artículo es reflexionar los elementos de mayor importancia dentro del contexto de la estrategia de la Gerencia del Capital Intelectual lo cual mediante un desarrollo de tipo documental, posibilito la referencia de importante autores en la materia. Dentro de los desarrollos presentados se tiene que la Gerencia del Capital Intelectual refiere a las acciones organizacionales para lograr el manejo inteligente de este recurso. De igual manera se destaca la importancia del mismo para el caso de las Universidades, como organizaciones facilitadoras de servicios, las cuales pueden asumir frente a los retos característicos del sistema, algunas determinaciones expresadas por los autores para la adecuada identificación, administración, establecimiento de indicadores así como de su preservación. Además se logró el análisis de los postulados de los más representativos autores en materia de capital intelectual, de igual manera la revisión de alguno modelos de trabajo que para el caso de las universidades resultan adecuados, destacándose los aportes de Bueno (2002), en su propuesta del Modelo de Capital Intelectual "INTELECT" (EUROFORUM). Así mismo resultó oportuno conocer la manera como las organizaciones gestionan los aspectos que permiten asegurar o preservar el capital intelectual de las mismas, permitiendo indicar algunas acciones viables para el caso de las Universidades.

Palabras clave: gerencia, capital intelectual, universidades, indicadores.

\section{ABSTRACT}

The purpose of this article of reflection, is to refer the most important elements in the context of the strategy of the Intellectual Capital Management which through a documentary-style development, enabled the authors important reference within which are: Drucker (2002), Edvinsson and Malone (1997), De la Fuente (2009), Ordonez (2002), Good (2002) among others. Among the developments have shown that the Intellectual Capital Management concerns organizational actions to achieve intelligent management of this resource. Similarly, it highlights the importance of the same for the case of universities, as facilitators of service organizations, which can take on the challenges facing the system characteristic, some determinations expressed by the authors for proper identification, management, establishment of indicators and their preservation. It also achieved the postulates analysis of the most representative authors in the field of intellectual capital, just as the review of any working models for the case of universities are appropriate, highlighting the contributions of Good (2002), in his proposed Model of Intellectual Capital "INTELECT" (EUROFORUM). So it was good to know the way organizations manage the aspects enabling secure or preserve the intellectual capital of the same, allowing viable indicate some actions for the case of universities.

Keywords: management, intellectual capital, universities, indicators.

\section{PROCESO DE GESTIÓN DEL CAPITAL INTELECTUAL}

Toda empresa organización tiene entre sus pertenencias una serie de bienes, con mayor o menor componente tangible o no, pero de reconocida importancia estratégica, siendo entre otros: el conocimiento del mercado; capacidades así como los recursos para el desarrollo de productos; componente humano poseedor de determinadas habilidades, actitudes,

\footnotetext{
Artículo de reflexión. Recibido el 14 de octubre de 2012. Aceptado noviembre 15 de 2012. Este artículo es resultado de las acciones mutuamente complementarias de la Red Autónoma de la Costa constituida por las Universidades Autónoma del Caribe y Universidad de la Costa.

"* Administrador de empresas, magister en gerencia de mercadeo. Correo: dsanz@cuc.edu.co

*** Administrador de empresas. Correo: rectoria@cuc.edu.co
} 
experiencias, conocimientos; reglas y políticas de trabajo; sistemas de información así como bases de datos específicas; relaciones con otras organizaciones y entidades.

Dichos ejemplos de bienes, son de compleja imitación pero forman parte del capital el cual permite a las organizaciones diferenciarse y construir ventajas competitivas. Dentro del ámbito de la literatura especializada se coincide en denominar a todos estos bienes como capital intelectual, y uno de los retos de las organizaciones es hacer que este capital se traduzca en factores que producen beneficios tangibles. Partiendo del hecho que las Instituciones de Educación Superior son estructuras operativas de producción, las cuales poseen una misión específica dentro del campo de la formación, es de entender que la misma basa su dinámica de acción a través del componente humano el cual motoriza cada una de sus unidades o departamentos. Por ello, e recurso humano es clave en el logro de sus objetivos, razón por la cual debe ser gestionado de manera inteligente.

Sin embargo, a pesar de que el capital intelectual adquiere este valor estratégico para las organizaciones del sector, el ámbito laboral aún se encuentra dominado por factores cotidianos de producción y, por ello, en ocasiones puede limitar la valoración del rendimiento humano el cual es determinante en todo sector.

En este orden de ideas, el proceso de gestión del capital intelectual implantado por Dow Chemical, presenta aspectos innovadores, como la creación del director general de gestión del activo ininteligible o un centro global de tecnología relacionada con la ininteligible, o el mantenimiento de canales abiertos de comunicación, de modo que todos trabajen en equipo.

Es indudable que no todas las organizaciones se sitúan alrededor de estas alternativas, aun cuando muchas de ellas aseguran estar integradas al proceso de gestión del capital intelectual, pero en el caso de las universidades, esto no sería de pensar, la operatividad del recurso humano es fundamental para el cumplimiento de las acciones estratégicas del sector.

Por ello, Balagué (2010), establece que es necesario que las universidades reconozcan sus fuentes de recursos así como las capacidades que contribuyen a lograr su autofinanciación, así como a elevar su imagen como institución: su producción científica e intelectual, el talento de sus miembros, su compromiso, su prestigio además su calidad tanto humana como profesional, y el soporte tecnológico que poseen para desarrollar sus conocimientos así como difundirlos.

En este particular, si se efectúa un análisis desde el punto de vista económico-financiero, el valor contable de la uni- versidad viene dado por la suma del valor de sus activos tangibles (edificios, equipos de laboratorio, ordenadores, entre otros), sin embargo, los grandes ausentes en los estados financieros, los intangibles, son los que en realidad aportan valor a las organizaciones, especialmente en aquellas que son intensivas en la creación y difusión del conocimiento. De acuerdo con Kaplan y Norton (1997), si estos elementos no se miden no se pueden gestionar, es como trabajar a ciegas.

A partir de este razonamiento, y teniendo presente el rol las universidades en la producción científica de la economía, es necesario proponer instrumentos de gestión que mejoren su aportación a la sociedad. Por ello, conformar programas orientados a la medición y gestión del capital intelectual representa una alternativa para potenciar la capacidad de logro de sus resultados, situación exigida para la sociedad del conocimiento, lo cual ha difundido en el ámbito académico la búsqueda de herramientas para medir así como gestionar al capital intelectual.

Sin embargo, en la actualidad no existe un consenso sobre cómo llevar a cabo tal medición y gestión, pero se han realizado diversos estudios que proponen alternativas tentadoras, a partir de las cuales se sustenta la metodología que se presenta para su implementación en las universidades. Previamente, se destacan algunos aspectos conceptuales generales sobre el capital intelectual. Ante esta realidad Marín (2001, 419), plantea:

El capital intelectual resulta de articular de manera ágil, dinámica y flexible los activos organizacionales que constituyen el capital humano, el capital estructural y el capital relacional. Al hablar de activos organizacionales se está haciendo referencia a aquella porción de los recursos de los que dispone la organización y que dinamizan los procesos que suceden dentro de la misma.

Por ello, para el caso de las universidades, el capital intelectual es el recurso capaz de generar dinamismo para la transformación, esta cualidad posee elementos que llevan a la obsolescencia, representada por la deficiente capacidad para soportar cambios contextuales. De allí, que es necesario canalizar la búsqueda del mejoramiento continuo dentro de los procesos y productos, a través del agregado permanente de valor. Esto se encuentra estrechamente relacionado con la capacidad de respuesta e innovación de las organizaciones frente a un ambiente competitivo.

De ahí que debe entenderse que el capital intelectual no tiene una expresión única ni está presente en un solo medio, esto se evidencia en el capital estructural, representado por todos los recursos de carácter físico, tecnológico y humano, los cuales se combinan de manera armónica con la finalidad de crear las condiciones para que los trabajadores 
del conocimiento, encuentren espacios de intersección, que se conviertan en plataforma de sustento, donde los insumos para producirlo se combinen para consolidar un conocimiento con cualidades para transferirlo en forma de capital, logrando una importante plataforma.

Una vez que las universidades internalizan la necesidad de cubrir el ciclo de generación de capital intelectual como su principal activo, es importante abordar un proceso formal de gestión el cual se corresponda con una visión de organización novedosa.

En este particular Drucker (2002) refiere

Una organización innovadora, que evidencie mecanismos de planificación, gerencia y evaluación garantizadores de optimizar el uso, promoción e integración de los diferentes recursos que propendan a la creación de espacios con alto sentido de pluralismo e interacción social, en razón de las funciones que cumplen como la producción y gestión de conocimiento.

Por lo tanto, las universidades están comprometidas en la búsqueda de la innovación en la gestión del capital intelectual, formalizando estructuras y asignando personal para el manejo de estos esfuerzos institucionales. En este sentido, Gates (2004) señala que la gestión del conocimiento ha de verse como una inversión en capital intelectual el cual conduzca con el tiempo hacia el logro de un capital intelectual, es decir que incremente la capacidad de las Universidades para concretar el mejor pensamiento y acción en el plano colectivo.

De esta forma se concibe la gestión de capital intelectual desde una dimensión amplia, que supera lo individual, para ingresar a los espacios institucionales, por ello, esta gestión es clave y debe incluirse en la filosofía y procesos de las universidades, a partir del reconocimiento de la necesidad de compartir información, producir y transferir el conocimiento, además resulta improrrogable por parte de estas organizaciones educativas asumir el reto.

Partiendo de los planteamientos de Meister $(1998,19)$ sobre universidades corporativas o empresariales, donde se enfatiza la creación de una infraestructura de sustento orientada a fortalecer y potencializar el capital humano, estructural, y relacional, y considerando las premisas referenciales indicadas, podría pensarse en un modelo descentralizado de tipo horizontal, en el cual la producción y transferencia del capital intelectual fluya a todos los actores organizacionales, de una manera mucho más rápida y efectiva.

\section{PROTECCIÓN DEL CAPITAL INTELECTUAL}

Al considerar este aspecto, no existe un criterio único y preciso que permita suponer al capital intelectual de una manera universal, sin embargo, Edvinsson y Malone (1999), refiere que es la posesión de conocimientos, experiencia aplicada, tecnología organizacional, relaciones con clientes y destrezas profesionales, que dan a una organización una ventaja competitiva.

Por su parte, Edvinsson (1997), lo refiere

Es como un árbol, hay una parte que es visible (las frutas) y una parte que está oculta (las raíces). Si solamente te preocupas por las frutas, el árbol puede morir. Para que el árbol crezca y continúe dando frutos, será necesario que las raíces estén sanas y nutridas. Si sólo nos concentramos en los frutos (los resultados financieros) e ignoramos los valores escondidos, la compañía no subsistirá en el largo plazo.

Según De la Fuente (2009), no cabe duda, de que se está en la era del conocimiento; dentro del ámbito empresarial, el conocimiento es cada vez más compartido además se encuentra en crecientemente actividad de distribución entre los miembros que la conforman. Así mismo, para el uso de la información, cada vez más organizaciones disponen de reglas corporativas claras sobre cómo, cuándo y para qué acceder utilizarla, lo cual determina una forma de protección.

Este aspecto es fundamental, debido a que si la información es un activo de la organización, el capital intelectual también lo representa, no se trata de establecer un rango de seguridad para las personas como elementos de propiedad por parte de la organización, se trata más bien de genera acciones que faciliten la preservación

De igual manera, De la Fuente (2009), considera que la totalidad de los activos corporativos son información confidencial de la cual sólo la empresa es legítima titular y que en la mayoría de los casos tiene una importancia crítica para la misma. Además, una gran cantidad de los activos que conforman el capital intelectual, están conformados por los derechos de la propiedad industrial o intelectual, secretos industriales o empresariales y know-how, en cualquier caso, la práctica totalidad de estos activos son información confidencial de la que sólo la empresa es legítima titular y que en la mayoría de las ocasiones tiene una importancia crítica para la misma.

En efecto, dentro de esa información confidencial, tiene espacio un borrador de memoria para una patente de invención, unos bocetos para el nuevo logo corporativo, planes financieros, información sobre nuevos productos, planes de externalización de áreas de negocio, entre otros aspectos, por lo cual no se diferencia de las realidades de las universidades las cuales también disponen de documentación que se caracteriza en este mismo ámbito. 
Es por ello, que se debe tener claro que las amenazas y riesgos para la organización y para la integridad de estos activos pueden ser tanto externas como internas. Para el caso de las internas, estas se derivan de la conducta del personal en el desarrollo de sus funciones así como en el uso que estos hacen de las herramientas tales como la navegación por internet, el correo electrónico, las herramientas informáticas y los dispositivos electrónicos que la organización pone a su disposición.

Al observar estas situaciones para el caso de las Universidades, las propias amenazas a encontrar en la red como virus, ataques de malware, gusanos de internet, además de posibles conductas de nuestros propios empleados tales como uso abusivo o impropio de medios informáticos, información confidencial, datos personales; amenazas, injurias, calumnias; infracción de derechos de propiedad intelectual e intelectual; utilización de activos de información con fines concurrentes.

Todo esto pueden situar a la organización en situación de grave riesgo con serias consecuencias para la misma desde el punto de vista de, las. fugas de información, tratamiento de datos personales, seguridad de los sistemas corporativos, reputación de la compañía y responsabilidad penal de las personas jurídicas, lo cual también afectaría en el caso de las universidades.

Es por ello por lo que procede articular los mecanismos oportunos dentro de la organización para prevenir y gestionar debidamente estos riesgos corporativos:

1. Respecto al Convenio Colectivo

2. Respecto al Contrato de Trabajo

3. Respecto a la Información Facilitada por los Empleados

4. Respecto a los Mecanismos de Control

\section{PONDERACIÓN Y DISEÑO DE INDICADORES}

Autores como Edvinsson y Malone (1999), consideran que al analizar a la gestión del conocimiento organizacional y al capital intelectual se determina la intangibilidad de ambos, de manera consecuente estos autores sostienen que la gestión del conocimiento organizacional abarca el capital Intelectual. Una definición acertada sobre capital intelectual, según Horta (2004), es aquella expresada como el conjunto de activos intangibles que posee una organización, estos activos pueden ser, recursos $\mathrm{o}$ actividades intangibles.

En un concepto más ampliose puede refier como un activo intangible que se corresponde con cualquier inversión de naturaleza intangible realizado por una empresa, se refleje o no en sus balances, pero también pueden ser el conjunto de saberes y competencias desarrolladas por esta. En términos generales, se afirma que identificar y medir el capital intelectual, tiene como objetivo convertir en visibles los intangibles ocultos, generadores de valor organizacional, por tanto los esfuerzos deben dirigirse a reflejarlos en un modelo representativo de la realidad social tal como puede ser el caso de las instituciones universitarias.

Para Rodríguez (2003), los indicadores de medición del capital intelectual son definidos como instrumentos de valoración de los activos intangibles de las organizaciones expresados en diferentes unidades de medida. Pueden ser de naturaleza cuantitativa o cualitativa, pero en el caso de las Universidades deben ser susceptibles de cuantificación.

A este respecto Martínez y Pérez, (2002), refieren que es interesante destacar que en la medición de algunos intangibles, como la eficiencia de las actividades de I+D, se ha distinguido entre indicadores basados en medidas objetivocuantitativas, indicadores basados en medidas subjetivocuantitativas e indicadores basados en medidas cualitativas. Los indicadores basados en medidas objetivo-cuantitativas son numéricos y toman como referencia información objetiva como la referida a los procesos organizativos.

Mientras que los indicadores basados en medidas subjetivocuantitativas proceden de valoraciones subjetivas (bueno, malo) a las que se asigna una equivalencia numérica. Finalmente, los indicadores basados en medidas cualitativas tienen en cuenta las opiniones o evaluaciones de expertos sin llegar a expresarlas de forma cuantitativa, además de lo complejo de esta trasformación.

No obstante, parece razonable pensar que si el cuadro de indicadores debe integrarse en el sistema de gestión de la empresa, cualquier medición cuantitativa debe expresarse de forma numérica. En cualquier caso, es importante destacar que los indicadores no son un objetivo en sí mismo, sino que son descripciones que requieren ser interpretadas y valoradas conforme a estándares.

En este sentido, Ordóñez de Pablos (2002), manifiesta que el capital intelectual es un elemento importante para crear una imagen holística de las empresas, es el valor oculto, que con su alta relevancia se reflejara por vía del descubrimiento del capital intelectual. Por su parte, Bueno (2002) quien define el capital intelectual como el conjunto de capacidades, destrezas y conocimientos de las personas que generan valor ya sea para las comunidades científicas, las universidades, las organizaciones y la sociedad en general.

En cuanto a Stewart (1997) hace su aporte teórico en el sentido de asemejar el capital intelectual al juego de valores intangibles que promueven la capacidad orgánica, así mismo, Bontis, Keow y Richardson (2000) corrobora cómo el C.I proporciona una diversidad de valor orgánico en las empresas generadoras de ganancia, y posicionamiento 
estratégico. Bajo este contexto se consideran empresas exitosas aquellas que, a manera de cultura corporativa, procuran aumentar el valor de su capital intelectual y en el futuro, lo cual resultad de interés para el caso de las universidades.

De acuerdo con estos autores referenciados, se puede concluir que, el valor del capital intelectual está centrado en aquellos individuos o grupos involucrados en la toma de decisiones del día a día que trabajan para simplificar y mejorar los flujos de datos, información y conocimiento y hacer organizaciones efectivas por medio de innovaciones, gracias a la maximización de rendimientos y la minimización de costos.

Luego de inspeccionar la conceptualización teórica del capital intelectual es pertinente hacer una revisión a los distintos modelos de capital intelectual relacionados con el tema y que sirven de columna vertebral al presente documento. Dichos modelos toman elementos generales, industriales y educativos; hechos que permiten establecer de manera tangencial, el modelo más apropiado para fundamentar algunos indicadores que se pueden presentar en las universidades

Con base en el anterior acercamiento conceptual, relacionado con los diferentes indicadores de Capital Intelectual aplicables a un modelo propio, es posible establecer el significado del Modelo de Capital Intelectual "INTELECT" (EUROFORUM), explicitado por Bueno (2002), quien con mayor facilidad presenta elementos y variables más aplicables en los ambientes de Capital Humano, Estructural y Relacional que vienen a configurarse en la razón de ser y del quehacer universitario; dados estos aspectos.

Decidir cómo invertir en las diferentes dimensiones de capital intelectual dependerá de la evaluación de las interdependencias entre ellas así como de la forma en que interactúan en las universidades. El hecho de que las mismas se refieran a aquel agente en el que radican, hace que normalmente existan asociaciones entre todas ellas de forma que unas son fuente de las otras.

Al respecto Edvinsson \& Malone (1997), señalan que la gestión del capital intelectual establece la utilización conjunta del capital humano, el capital estructural y el capital relacional, incrementando de este modo la interacción entre sus componentes. Ordóñez de Pablos (2004), analiza las interrelaciones entre los diferentes componentes del capital intelectual -humano, relacional y estructural; y sus resultados muestran que el capital humano tiene un efecto significativo sobre el desarrollo del capital relacional y el capital estructural, además que el capital relacional muestra un efecto significativo sobre el capital estructural.
Por su parte Camisón et al. (2000) indican que un incremento en el capital humano de la organización contribuye en la mejora de las relaciones entre los empleados, los directivos, los clientes, los proveedores, entre otros. Por consiguiente es posible esperar un efecto del capital humano sobre el capital relacional, y sobre el capital estructural. Este es un aspecto que resultad de interés para el caso de las universidades debido a la gama de impactos positivos que se logran a través de una manejo inteligente del capital intelectual, por lo cual gestionar su medición resulta una práctica productiva y no una formalidad para la gerencia.

Por su parte, Bontis (2000) y Wang \& Chang (2005), expresan que existe una interrelación entre los distintos capitales, de ahí que el capital humano afecta directamente al de innovación y al de procesos y en resumen, es el factor primario líder en el cual la gestión de conocimientos debe poner su máximo esfuerzo. En el caso de Argyris \& Schön (1978), Senge (1990) y Nonaka (1991), estos plantean que el capital humano y el capital relacional constituyen la base de toda la creación de conocimiento en las organizaciones.

Sin embargo, si se desea protegerlo de las amenazas que suponen el abandono de la organización por parte de empleados clave y la pérdida de relaciones, deben institucionalizarlo en rutinas, cultura, estructuras, y de esta forma, utilizar en toda la organización este conocimiento representado en el capital estructural. Esta es una excelente estrategia para el caso de las universidades, en procura de la protección del capital intelectual.

Así, aunque Bontis (1998) y Bueno (2002), sugieren que la fuente del capital intelectual está en las personas y por lo tanto el capital humano es la base para la generación de las otras dos dimensiones de ese capital, también es cierto que una mayor aportación de capital social -estructural y relacional- genera un incremento en el capital humano.

\section{REFERENCIAS}

Argyris, C., \& Schön, D. A. (1978): Organizational Learning: A Theory of Action. Perspective. Massachusetts: AddisonWesley.

Balagué J. (2010): La medición del capital intelectual en las universidades. Un modelo para potenciar su aportación a la sociedad. Revista Capital Humano, № 185, Sección Artículos.

Bontis, N. (1998). Intellectual capital: an exploratory study that develops measures and models. Management decision $36(2), 63-76$ 
Bontis, N., WCC Keow, S Richardson (2000). Intellectual capital and business performance in Malaysian industries. Journal of intellectual capital 1 (1), 85-100

Bueno E. (2002): Capital intelectual y producción científica, Dirección General de Investigación, Consejería de Educación, Comunidad de Madrid.

Bueno E. (2000) Modelo de dirección estratégica por competencia: el capital intangible. Madrid, Euroforum.

Camisón, C.; Palacios, D.; Devece, C. (2000): Un nuevo modelo para la medición de capital intelectual: el modelo Nova. X Congreso Nacional CEDE. Oviedo.

De la Fuente (2009): La Protección del Capital Intelectual Corporativo. Santiago: Editorial WRK.

Drucker P. (2002): Gerencia para el futuro. Bogotá: Norma.

Edvinssion, L. (1997). Developing intellectual capital at Skandia, Long Range Panning, 30 (June); pp.366-373.

Edvinsson L. y Malone M. (1999): El Capital Intelectual: Cómo identificary calcular el valor inexplorado de los recursos intangibles de su empresa. (2nd ed., pp. 30-48). Bogotá: Norma.

Horta R. (2004): Informes de Capital Intelectual y Responsabilidad Social Corporativa. Revista FCE. Revista electrónica de la Faculta de Ciencias Empresariales de la Universidad Católica. Uruguay. En: http://www.ucu.edu.uy/Facultades/ CienciasEmpresariales/RevistaFCE/revista3/pdf/horta.pdf

Kaplan R. y Norton D. (1997): Cuadro de Mando Integral, The Balanced Scorecard. Madrid: Gestión.
Marín, F. (2001): El capital intelectual como activo organizacional. Espacio abierto, vol. 10, núm. 3, julio septiembre.

Martínez C. y Pérez E. (2002): "Teoría Avanzada de Organización y Gestión”: Del Management a la teoría avanzada de la Gestión, Modelo PODER-SEEO. (2nd ed., pp. 48-51). Bogotá D.C.: Editorial Universidad Nacional de Colombia.

Nonaka I. (1991) The knowledge-creating company. Harvard Business Review, 96-104.

Osorio, M. (2003): El Capital Intelectual en la gestión del conocimiento. ACIMED, V.11 No.6, Cuba, nov.-dic. 2003

Ordoñez de Pablos, P. (2004): Capital intelectual, gestión del conocimiento y sistemas de gestión de recursos humanos: influencia sobre los resultados organizativos en la industria manufacturera española. XIV Congreso ACEDE.

Rodríguez O. (2003): Indicadores de capital intelectual: concepto y elaboración. Madrid: Instituto Universitario de Administración de Empresas. Universidad Autónoma de Madrid.

Stewart T. (1997): Intellectual Capital: The New Wealth of Organizations. New York, NY: Doubleday Currency.

Senge, P. M. (1990). The fifth discipline: The art and practice of the learning organization. New York: Doubleday Currency.

Wang, W.; Chang, C. (2005): Intellectual capital and performance in causal models. Evidence from the information technology industry in Taiwan. Journal of Intellectual Capital, 6(2): 222-236. 